\title{
Nephrotic Syndrome, CTCAE
}

National Cancer Institute

\section{Source}

National Cancer Institute. Nephrotic Syndrome, CT CAE. NCI Thesaurus. Code C143239.

A disorder characterized by symptoms that include severe edema, proteinuria, and

hypoalbuminemia; it is indicative of renal dysfunction. 